\title{
EsDREB2B, a novel truncated DREB2-type transcription factor in the desert legume Eremosparton songoricum, enhances tolerance to multiple abiotic stresses in yeast and transgenic tobacco
}

Xiaoshuang $\mathrm{Li}^{1,2,3}$, Daoyuan Zhang ${ }^{1 *}$, Haiyan $\mathrm{Li}^{1,2}$, Yucheng Wang ${ }^{1}$, Yuanming Zhang ${ }^{1}$ and Andrew J Wood ${ }^{3}$

\begin{abstract}
Background: Dehydration-Responsive Element-Binding Protein2 (DREB2) is a transcriptional factor which regulates the expression of several stress-inducible genes. DREB2-type proteins are particularly important in plant responses to drought, salt and heat. DREB2 genes have been identified and characterized in a variety of plants, and DREB2 genes are promising candidate genes for the improvement of stress tolerance in plants. However, little is known about these genes in plants adapted to water-limiting environments.

Results: In this study, we describe the characterization of EsDREB2B, a novel DREB2B gene identified from the desert plant Eremosparton songoricum. Phylogenetic analysis and motif prediction indicate that ESDREB2B encodes a truncated DREB2 polypeptide that belongs to a legume-specific DREB2 group. In E. songoricum, ESDREB2B transcript accumulation was induced by a variety of abiotic stresses, including drought, salinity, cold, heat, heavy metal, mechanical wounding, oxidative stress and exogenous abscisic acid (ABA) treatment. Consistent with the predicted role as a transcription factor, ESDREB2B was targeted to the nucleus of onion epidermal cells and exhibited transactivation activity of a GAL4-containing reporter gene in yeast. In transgenic yeast, overexpression of EsDREB2B increased tolerance to multiple abiotic stresses. Our findings indicate that EsDREB2B can enhance stress tolerance in other plant species. Heterologous expression of ESDREB2B in tobacco showed improved tolerance to multiple abiotic stresses, and the transgenic plants exhibited no reduction in foliar growth. We observed that EsDREB2B is a functional DREB2-orthologue able to influence the physiological and biochemical response of transgenic tobacco to stress.
\end{abstract}

Conclusions: Based upon these findings, EsDREB2B encodes an abiotic stress-inducible, transcription factor which confers abiotic stress-tolerance in yeast and transgenic tobacco.

Keywords: Cold, DREB, Drought, Heat, Proline, Salt, Stress, Transcript accumulation, Transgenic tobacco

\footnotetext{
* Correspondence: zhangdy@ms.xib.ac.cn

'Key Laboratory of Biogeography and Bioresource in Arid Land, Xinjiang Institute of Ecology and Geography, Chinese Academy of Sciences, Xinjiang Urumqi 830011, China

Full list of author information is available at the end of the article
} 


\section{Background}

Abiotic stresses such as drought, salinity, and extreme temperature negatively impact the growth and productivity of crop plants [1,2]. Among these various stresses, drought is the primary factor causing crops to lose productivity [3]. The impacts of drought are likely to become even more pronounced as desertification and global climate change progress around the world. Plants have evolved a diverse set of morphological, biochemical, physiological and molecular adaptations to both survive and sustain growth in harsh environments [4]. Understanding drought response mechanisms is essential to improving drought tolerance and reducing the influence of drought on plant growth and crop yield.

Stress-associated genes and proteins for each of the steps downstream of the drought response can be grouped into two major categories: single functional genes and transcription factors (TFs) [5]. TFs are critical regulators of the changes in gene expression used to respond to environmental stress [6]. A single TF can control the expression of many downstream genes by binding to the cis-acting element in the promoters of the target genes [7]. Current efforts to improve plant stress tolerances by the genetic transformation of TF genes have resulted in several important achievements. The DREB (dehydration-responsive element binding proteins) gene family broadly participates in plant stress response pathways [8]. The DREB2 gene family is particularly important in the responses to dehydration and heat stress [9].

DREB homologs have been identified in a wide variety of plants, including grasses [10-13], fruits [14,15], vegetables [16,17], crops [18-23] and Arabidopsis [24]. Over the past decade, many reports, both in the laboratory and the field, have demonstrated that DREBs hold great potential for improving the stress tolerance of plants in response to drought, salt, cold, and heat [25-28]. Consequently, DREB genes are promising candidates for genetic engineering and have been extensively investigated in recent years. Characterization of DREB/ERF genes from wild species (i.e. non-cultivated and nonmodel plants) may provide novel homologues for breeding stress resistance into crops [9]. Although primarily cloned from herbaceous plants, DREB homologues have been characterized in woody, desert plants such as Populus euphratica [29] and Caragana korshinskii [30]. Analysis of naturally stress-tolerant plants proffers novel stress-associated gene resources and may enhance our knowledge of DREB expression and function.

Eremosparton songoricum (Litv.) Vass. is a leafless perennial dwarf shrub endemic to central Asia [31]. E. songoricum is adapted to a harsh desert environment and is a wild pioneer species used to protect the local ecosystem from desertification in the Gurbantunggut desert of Xinjiang. This extremely drought-tolerant leguminous shrub is being developed as a model organism for investigating the morphological, biochemical, physiological and molecular adaptations to a desert environment $[32,33]$. We report here the cloning of a drought-induced $D R E B$ gene from E. songoricum (EsDREB2B). We have characterized EsDREB2B gene expression, subcellular localization, and transactivation activity. In addition, we have investigated the function of $E s D R E B 2 B$ in transgenic yeast and tobacco and evaluated transgenic plant tolerance to a suite of abiotic stresses.

\section{Results}

\section{Characterization of EsDREB2B from E. songoricum}

A cDNA clone encoding a DREB2 protein was isolated from two-week-old $E$. songoricum seedlings exposed to $20 \%(\mathrm{w} / \mathrm{v})$ PEG for $12 \mathrm{~h}$. For clarity and in keeping with the nomenclature established for this gene family in other plants, we have designated the gene EsDREB2B and the encoded protein EsDREB2B. EsDREB2B [GenBank: HQ687367] was 757 bp in length and contained a single, continuous open reading frame (ORF). The ORF encoded a polypeptide of 207 amino acids with a predicted molecular mass of $22 \mathrm{kDa}$ and a predicted $\mathrm{pI}$ of 8.6. The deduced $E$. songoricum polypeptide has significant sequence identity to the conserved AP2/ERF DNA-binding domain [34] and is more than $80 \%$ identical to CAP2 from chickpea [21] (Figure 1A). Significant amino acid sequence similarity is also seen with DREB2B deduced polypeptides from soybean (78\%), rice (42\%) and Arabidopsis (45\%). Alignment of the AP2/ERF DNA-binding domain demonstrates that EsDREB2B contains the conserved valine (V14) and glutamic acid (E19) residues observed in other DREB predicted polypeptides (Figure 1A) [35,36]. Comparison of the pairwise alignments was conducted using ClustalX 2.1. To determine the architecture of the EsDREB2B gene, E. songoricum g DNA was amplified using cDNA-specific primers. EsDREB2B is an intron-less gene similar to $D R E B$ genes reported for Salicornia brachiate (SbDREB2A) [37], chickpea (CAP2) [21] and soybean (GmDREB2Band GmDREB2C) [35].

EsDREB2B is a member of the AP2/ERF protein family and belongs to the "Group IV, A-2" sub-group [34]. Other members of this sub-group include CAP2 [21], GmDREB2B [35], OsDREB2B [38] and AtDREB2A [24]. Deduced polypeptides of this sub-group each contain single copy of the motifs CMIV-1, CMIV-2 and AP2, and may contain a single copy of CMIV-3 (Figure 1B). CMIV-1 is 27 amino acid residues and defined by the conserved amino acid motif [K/R]GKGGPXN. CMIV-2 is 29 amino acids residues, and CMIV-3 is 25 amino acids residues. CMIV-1 and CMIV-2 are predicted to contain a nuclear localization sequence (NLS) $[24,39,40]$. EsDREB2B contains a newly identified motif CMIV-4 (Additional file 1: Figure S1). The entire predicted 


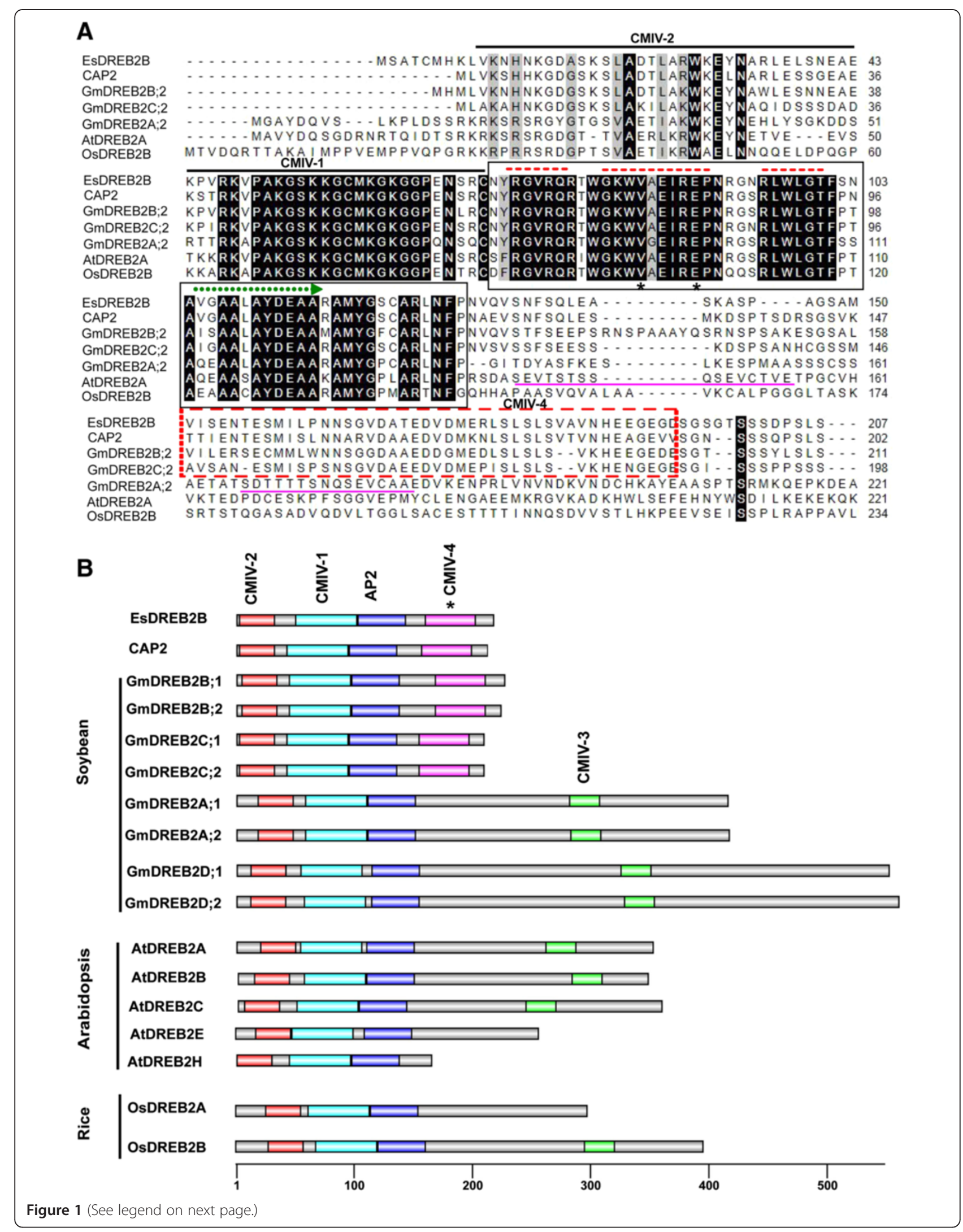




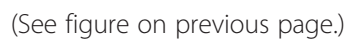

Figure 1 Alignment and schematic diagram of EsDREB2B and homolog proteins. A Alignment of the predicted EsDREB2B amino acid sequence with 6 typical DREB2-type proteins from chickpea, soybean, Arabidopsis and rice. Alignment of the predicted polypeptide sequences (residues 1-210) was performed using ClustalX2.1 software. Black and light gray shading indicate identical and conserved amino acid residues. The single lines in black and pink represent predicted motifs (CMIV-1 and CMIV-2) and core negative regulation domain (NRD) of AtDREB2A and GmDREB2A;2 proteins, respectively. The AP2 domain is boxed in black and the three $\beta$ sheets regions are dash lined in red, the a helix region was labeled in green dash line arrow. Asterisks represent the V14 and E19 conserved amino acids of AP2 domain. The truncated specific CMIV-4 motif was boxed in red. The protein accession numbers are as follows: EsDREB2B (ADV57357.1), CAP2 (ABC49682.1), GmDREB2A;2 (AFU35563.1), GmDREB2B;2 (AAT12423.1), GmDREB2C;2 (AAP83131.1), AtDREB2A (AAL36328.1), OsDREB2B (NP_001055260.2). B Architectures of conserved protein motifs. 17 subtype 1 (DREB2-type) transcriptions factors including E. songoricum, chickpea, soybean, Arabidopsis and rice are collected for motif detection using MEME online tools. Parameters are as follows: 0 or 1 occurrence of a single motif per sequence, motif width ranges of 6 to 50 amino acids, and 10 as the maximum number of motif. CMIV-1(cyan), CMIV-2 (red) and CMIV-3 (green) motifs are reported by Nakano et al. (2006). The AP2 domain (blue) indicate the conserved AP2/ERF DNA-binding domain, the CMIV-4 conserved motif (pink and marked with*) indicate a specific motif shared with truncated DREB2-type proteins. The domains are drawn to scale. AtDREB2A (AAL36328.1), AtDREB2B (AAP37710.1), AtDREB2C (NP_565929.1), AtDREB2E (NP_181368.1), AtDREB2H (NP_181566.2) are from Arabidopsis; GmDREB2A;1 (AFU35562.1), GmDREB2A;2 (AFU35563.1), GmDREB2B;1 (AAQ57226.1), GmDREB2B;2 (AAT12423.1), GmDREB2C;1 (NP_001236509.1), GmDREB2C;2 (AAP83131.1), GmDREB2D;1 (AFU35564.1), GmDREB2D;2 (AFU35565.1) are from soybean; OsDREB2A (NP_001042107.1) and OsDREB2B (NP_001055260.2) are from rice.

polypeptide EsDREB2B was aligned and compared to 30 DREB2 sequences including AtDREB2A and OsDREB2A (Figure 2). Phylogenetic analysis shows a well-supported group of DREB2 sequences derived from leguminous plants (Fabaceae) including EsDREB2B, CAP2 and GmDREB2B. Additionally, the Fabaceae group contains two wellsupported subgroups designated "Legume 2B" and "Legume 2C". Similar gene tree architectures were obtained using only the AP2 domain, and alternate analytical models for tree construction (Additional file 2: Figure S2).

These legume-specific deduced polypeptide sequences contain canonical DREB2 motifs including the AP2 domain and the CMIV-1 and CMIV-2 motifs [22,34,38]; however, the legume-specific ORFs are truncated (i.e. less than 220 amino acid residues) relative to sequences derived from soybean [35], rice [38] and Arabidopsis [24], lack CMIV-3 and contain a novel motif CMIV-4 (Figure 1). CMIV-4 is 45 amino acid residues and defined by the conserved amino acid motif NSG[V/G] DAX[E/D]D[V/D][D/G]MXX[L/T]SLSL[S/T]V. CMIV-4 is present in each of the truncated DREB2 sequences from legumes, and no other deduced polypeptide sequences in the databases share significant homology with this motif. EsDREB2B and related sequences do not contain a negative regulatory domain (NRD) $[22,38,40]$. The NRD is present in AtDREB2A, AtDREB2B and AtDREB2C and GmDREB2A;2 [22], and is located immediately downstream of the AP2 domain. Deletion of the NRD in AtDREB2A transforms the protein to a constitutively active form [39].

\section{Analysis of EsDREB2B expression by qRT-PCR in $E$. songoricum seedlings}

Quantitative RT-PCR (qRT-PCR) was employed to analyze the steady-state transcript accumulation of EsDREB2B in $E$. songoricum seedlings (Figure 3). EsDREB2B transcript was detectable in roots, stem and leaves of unstressed (i.e. control) seedlings, and was most abundant in leaves (Additional file 3: Figure S3). EsDREB2B transcript abundance was measured in plants exposed to osmotic stress (20\% PEG), salinity (250 mM NaCl), low temperature $\left(4^{\circ} \mathrm{C}\right)$, exogenous ABA $(100 \mu \mathrm{M} \mathrm{ABA})$, elevated temperature $\left(42^{\circ} \mathrm{C}\right)$, oxidative stress $\left(50 \mathrm{mM} \mathrm{H}_{2} \mathrm{O}_{2}\right)$ and heavy metal $\left(0.5 \mathrm{mM} \mathrm{CuSO}_{4}\right)$ (Figure 3$)$. In response to each treatment, EsDREB2B steady-state transcript amounts increased, reached a peak at either $6 \mathrm{~h}$ or $12 \mathrm{~h}$ and subsequently declined at $24 \mathrm{~h}$ (Figure 3 ). With the exception of exogenous ABA exposure and cold stress, EsDREB2B transcript was more abundant at $24 \mathrm{~h}$ relative to the control (i.e. 0 h). EsDREB2B steady-state transcript amounts increased more than 20-fold in response to elevated temperature (Figure 3B). These results suggest that EsDREB2B is widely involved in multiple stress responses, and may function in the ABA-mediated stress tolerance pathway.

\section{Subcellular localization and transactivation analysis of EsDREB2B protein}

The AP2/ERF family members function as transcription factors in plants and have been documented to localize to the nucleus of Arabidopsis protoplasts [22] and onion epidermal cells $[12,41]$. The subcellular location of the EsDREB2B protein was analyzed by the transient expression of a GFP fusion protein introduced into onion epidermal cells (Figure 4A). The control protein (p35S-GFP) was distributed in both the cytoplasm and the nucleus of the onion cells, while the EsDREB2B fusion protein (p35S-EsDREB2B: GFP) was only detectable in the nucleus (Figure 4A). The transcriptional activation ability of EsDREB2B was examined using the yeast one-hybrid assay. Yeast cells transfected with the pBridge-BD vector acted as the negative control and were unable to grow on the SD medium without His and Trp (SD/-His-Trp) (Figure 4B). However, the cells harboring 


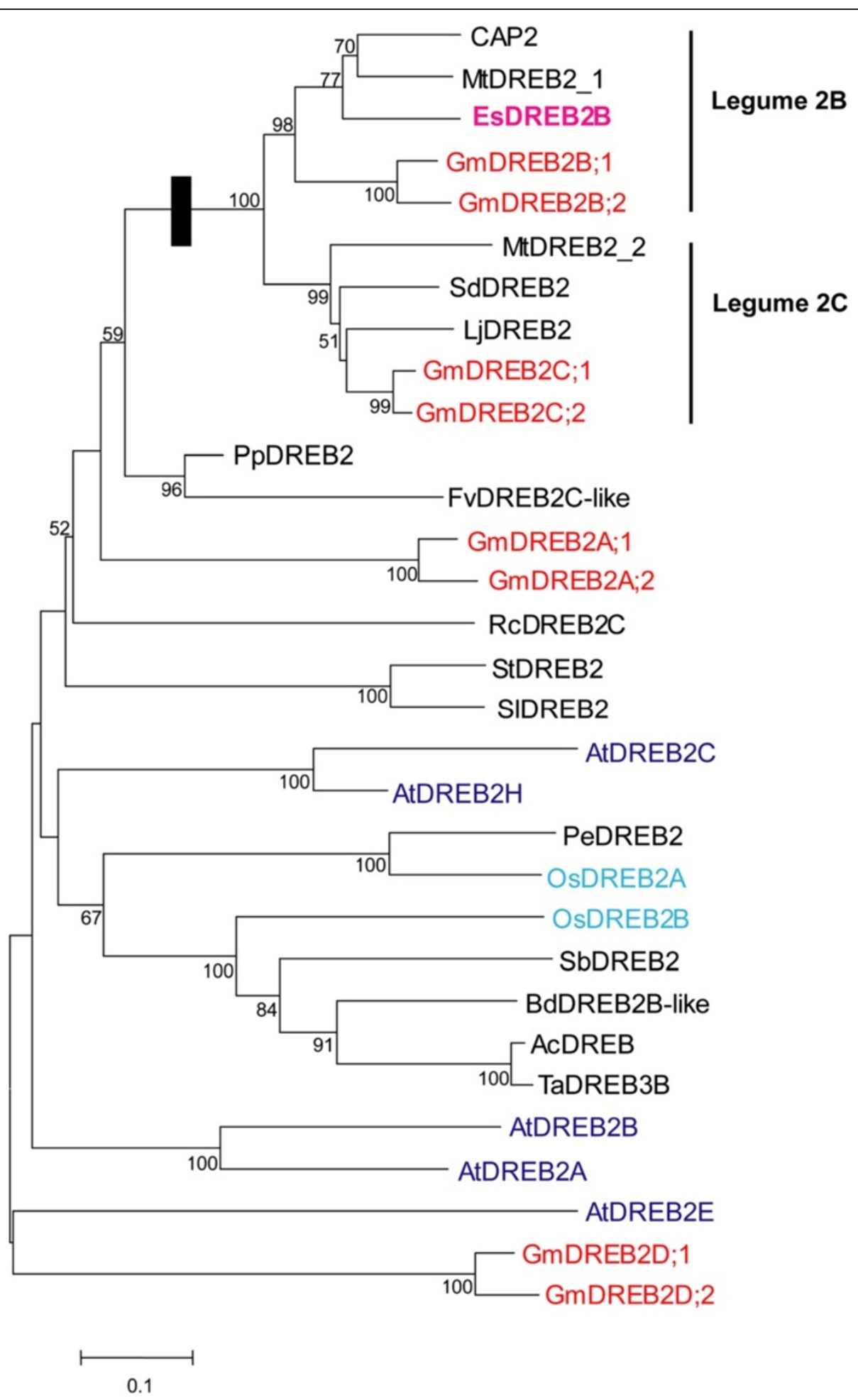

Figure 2 (See legend on next page.) 
(See figure on previous page.)

Figure 2 Phylogenetic analysis of EsDREB2B and homologous proteins. Phylogenetic tree of subtype1 (DREB2-type) transcription factors in E. songoricum (EsDREB2B, pink and bold), soybean (red), Arabidopsis (blue), rice (cyan) and other deduced polypeptides with high sequence identities with EsDREB2B. The neighbor-joining tree is based on an alignment of the complete deduced polypeptide sequences (residues 1-210). Bootstrap values from 1000 replicates were used to assess the robustness of the tree. Solid box ( $\mathbf{m})$ indicated truncated sequences clade with CMIV-4 motif. Bootstrap values $>50$ are shown. EsDREB2B (ADV57357.1) is from E. songoricum; CAP2 (ABC49682.1) is from chickpea; AtDREB2A (AAL36328.1), AtDREB2B (AAP37710.1), AtDREB2C (NP_565929.1), AtDREB2E (NP_181368.1), AtDREB2H (NP_181566.2) are from Arabidopsis; GmDREB2A;1 (AFU35562.1), GmDREB2A;2 (AFU35563.1), GmDREB2B;1 (AAQ57226.1), GmDREB2B;2 (AAT12423.1), GmDREB2C;1 (NP_001236509.1), GmDREB2C;2 (AAP83131.1), GmDREB2D;1 (AFU35564.1), GmDREB2D;2 (AFU35565.1) are from soybean; OsDREB2A (NP_001042107.1) and OsDREB2B (NP_001055260.2) are from rice; in addition, other protein accession numbers are as follows: EsDREB2B (ADV57357.1); MtDREB2B (AFK38091.1); CAP2 (ABC49682.1); MtDREB2C (XP_003597059.1); LjDREB2C (AFK47592.1); SdDREB2C (AFP89590.1); PpDREB2C-like (EMJ17611.1); FVDREB2C-like (XP_004293490.1); StDREB (AEI98833.1); SIDREB (NP_001234689.1); RCDREB2C (XP_002520794.1); PeDREB2 (ABY19375.1); SbDREB2 (AFI71292.1); BdDREB2B-like (XP_003568655.1); AcDREB (ACX94337.1); TaDREB3B (AAX13278.1). Blue $=$ A. thaliana, Red = G. $\max$, Turqoise $=$ O. sativum and Purple $=$ E. songoricum.
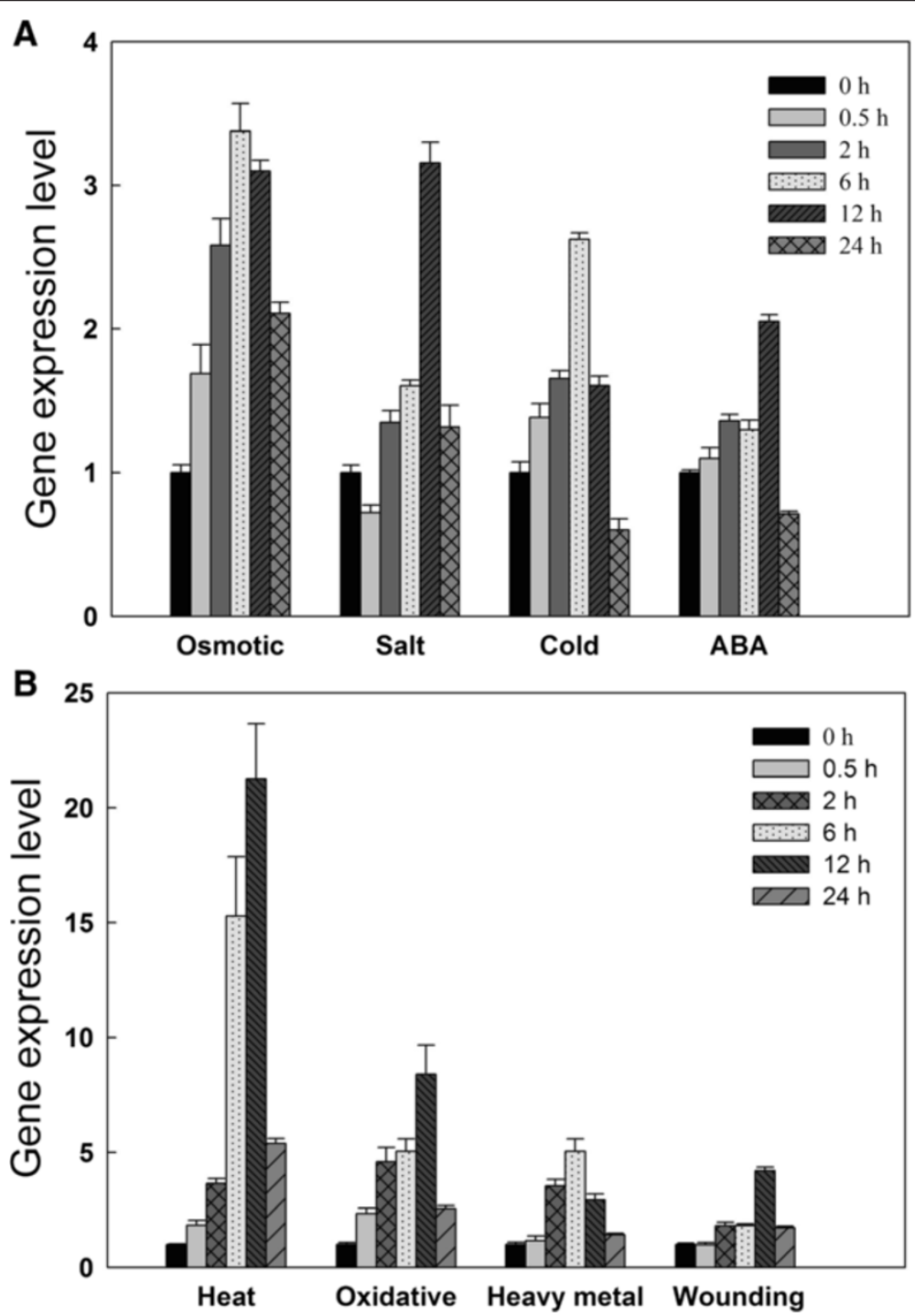

Figure 3 Expression patterns of EsDREB2B gene in response to various stress treatments. EsDREB2B expression in two-week old $E$. songoricum seedlings with $\mathbf{A}$ osmotic, salt, cold stresses and ABA treatment; $\mathbf{B}$ heat, oxidative, wounding and heavy metal stresses; The relative gene expression level of ESDREB2B gene were calculated relative to $0 \mathrm{~h}$ under corresponding stress treatment using $2^{-\triangle \Delta C T}$ method. Error bars indicate SD $(n=3)$. 


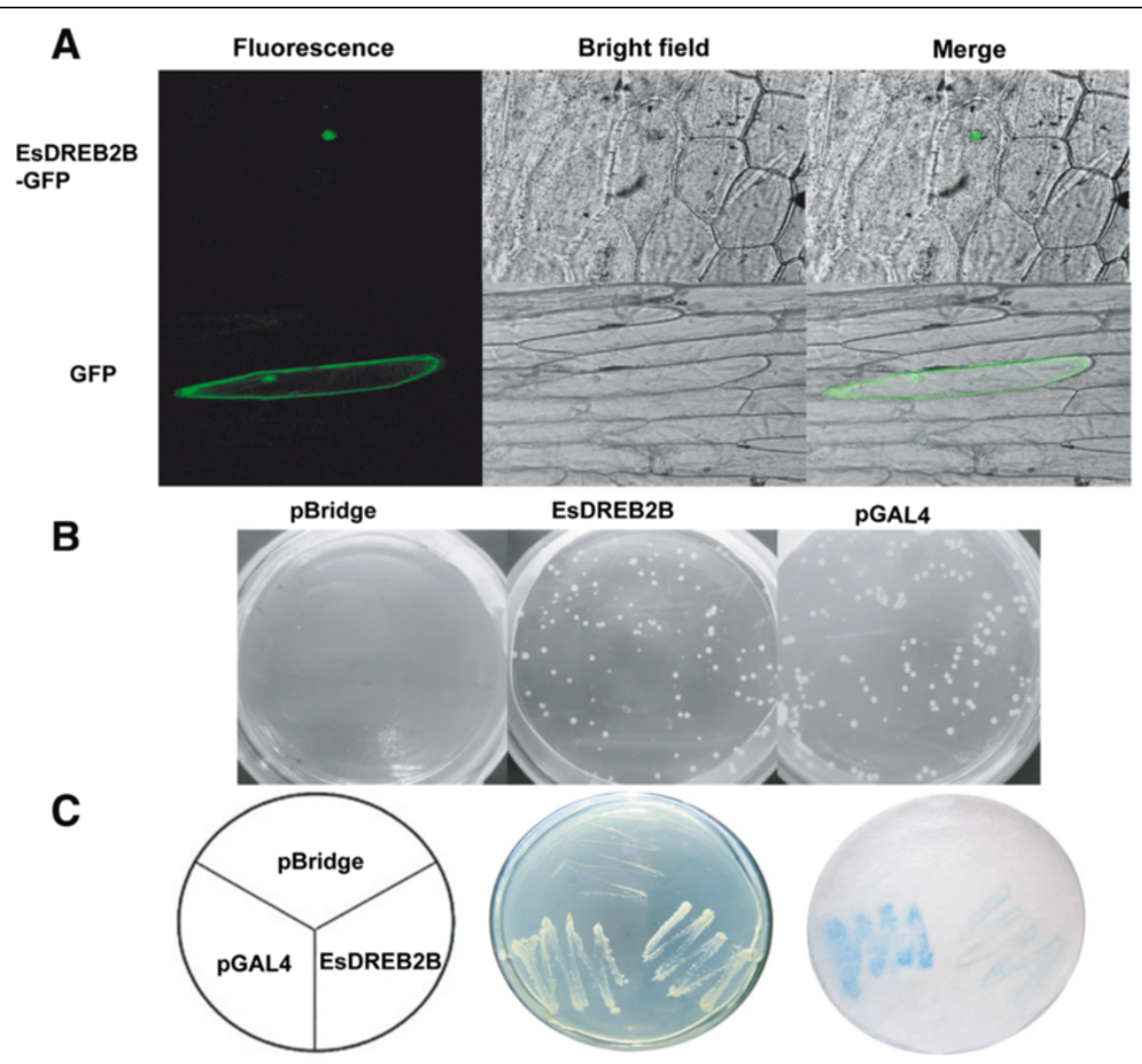

Figure 4 Subcellular localization and transactivation analysis of EsDREB2B protein. A GFP and EsDREB2B: GFP fusion protein were transiently expressed under the control of the CaMV 355 promoter in onion epidermal cells and observed with a laser scanning confocal microscope. The images were presented fluorescence, bright field and merge of bright field and fluorescence. B The transcriptional activation ability of EsDREB2B was examined using the yeast one-hybrid assay. The growth of yeast cells on SD/-His-Trp medium and $\mathbf{C} \beta$-galactosidase activity was analyzed. PBridge-BD vector served as the negative control and pBridge-BD-GAL4 served as the positive control.

pBridge-BD-EsDREB2B or pBridge-BD-GAL4 (the positive control) grew well on the SD/-His-Trp medium (Figure 4B), and these cells turned blue using the $\beta$-galactosidase activity assay (Figure $4 \mathrm{C}$ ). These results indicate that EsDREB2B was targeted to the nucleus and exhibited transactivation activity of a GAL4-containing reporter gene. Similar results have been obtained for CAP2 [21] and LcDREB [12].

\section{EsDREB2B protein expression in transgenic yeast enhances tolerance to multiple stresses}

DREB2 transcriptional factors have not been extensively studied in yeast (Saccharomyces cerevisiae). CAP2 has been demonstrated to provide enhanced tolerance to elevated temperature $\left(39^{\circ} \mathrm{C}\right)$ in yeast [42]. The EsDREB2B cDNA, driven by a galactose-inducible promoter (pYES2), was introduced into S. cerevisiae (INVSc1) in order to investigate the ability of EsDREB2B to enhance abiotic stress tolerance (Figure 5). Semi-quantitative RT-PCR analysis confirmed that EsDREB2B was expressed in yeast with a peak expression level occurring at $36 \mathrm{~h}$ (Additional file 4:
Figure S4). Subsequently, all yeast cells were evaluated after $36 \mathrm{~h}$ exposure to stress. The growth rate of EsDREB2Btransformed S. cerevisiae (pYES2-EsDREB2B) was similar to the growth rate of empty vector controls (pYES2) under non-stress conditions (Figure 5A). S. cerevisiae was able to grow in the presence of $30 \%$ (w/v) PEG (Figure 5B), $5 \mathrm{M}$ $\mathrm{NaCl}$ (Figure 5C), at low temperature (Figure 5D) or elevated temperature (Figure $5 \mathrm{E}$ ), or in the presence of $20 \mathrm{mM} \mathrm{H}_{2} \mathrm{O}_{2}$ (Figure 5F). EsDREB2B-transformed S. cerevisiae had dramatically enhanced growth relative to the control yeast in the presence of PEG (Figure 5B), $\mathrm{NaCl}$ (Figure 5C) and heat (Figure 5E). These results indicated that EsDREB2B was functional in yeast cells and improved the tolerance to PEG-, salinity- and heat-stress.

\section{Evaluation of EsDREB2B in transgenic tobacco in response} to drought, salt, cold and heat

Since EsDREB2B transcript accumulation was up-regulated by a number of abiotic stresses (Figure 3 ) and enhanced the stress tolerance of yeast (Figure 5), transgenic Nicotiana tobacco plants containing the EsDREB2B cDNA were 


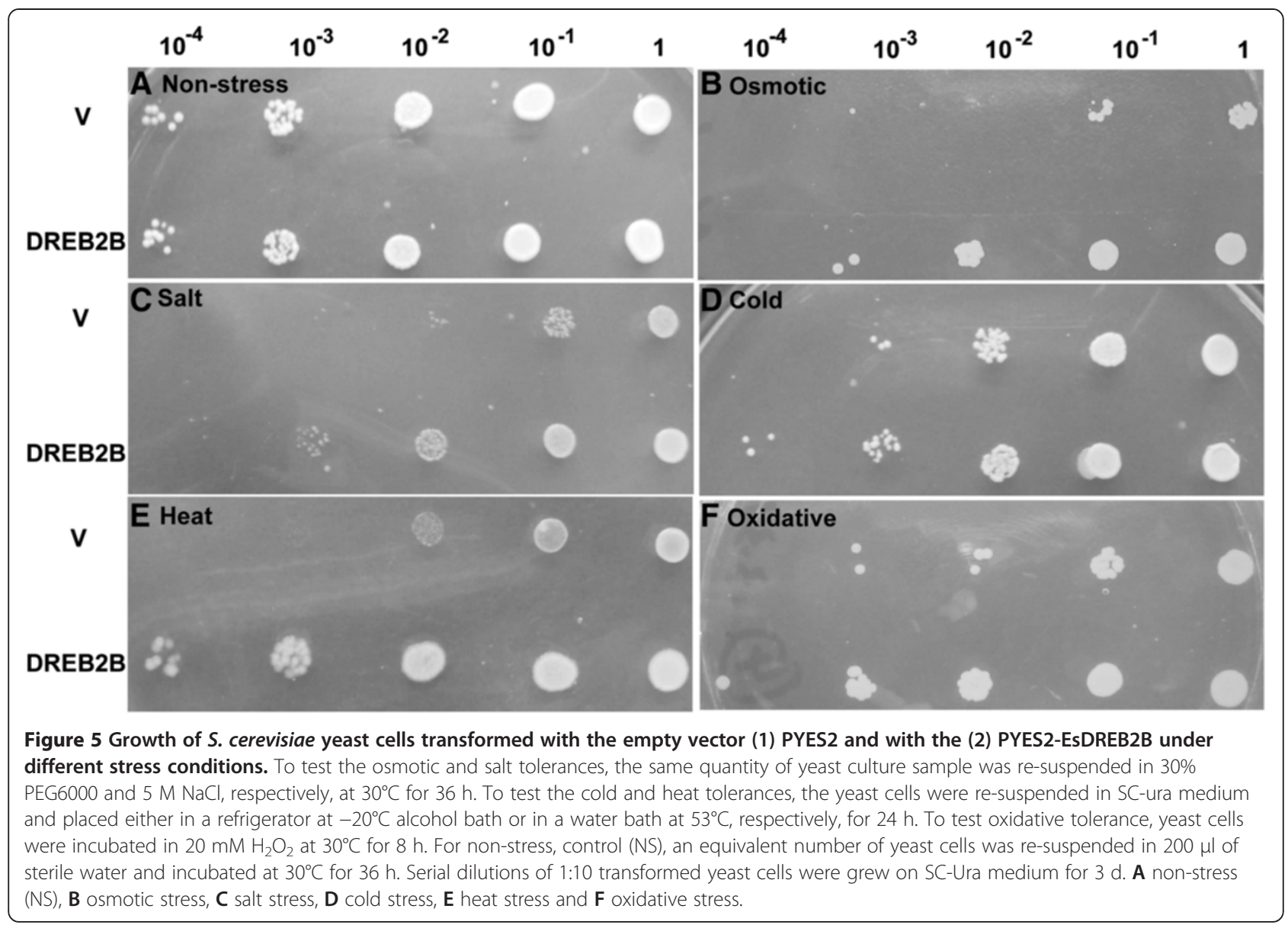

generated to evaluate whether overexpression of the ORF could enhance stress tolerance. Hygromycin-resistant transgenic lines were confirmed by PCR and RT-PCR amplification using gene specific primers (data not shown). L1 and L2 were two, randomly selected, independent transgenic lines that overexpressed EsDREB2B. For osmotic- and salinity-stress, two-week-old seedlings were grown on MS medium and transplanted to MS medium supplemented with $200 \mathrm{mM}$ mannitol or $200 \mathrm{mM} \mathrm{NaCl}$ for $20 \mathrm{~d}$. For low and elevated temperature-stress, two-week-old seedlings grown in MS medium, transplanted to MS medium and incubated for $20 \mathrm{~d}$ at $4^{\circ} \mathrm{C}$ or $7 \mathrm{~d}$ at $42^{\circ} \mathrm{C}$, respectively. EsDREB2B transgenic tobacco grew normally when unstressed, and the non-transformed and transgenic lines showed no difference in the fresh weight under non-stress growth conditions (Figure 6A). The fresh weight of WT plants was significantly lower in response to all four treatments (Figure 6A). Transgenic plants maintained significantly higher fresh weight in all four treatments. And with the exception of elevated temperature, the salt and coldstress L2 transgenic fresh weight values were more similar to unstressed values. Only the osmotic stressed L1 transgenic fresh weight was similar to the unstressed samples. After $20 \mathrm{~d}$ of mannitol treatment, dark green leaves with curled edges were observed in both the transgenic and WT plants (Additional file 5: Figure S5). After $20 \mathrm{~d}$ of salt treatment, yellow leaves were observed on all of the plants. After $20 \mathrm{~d}$ of cold exposure, both WT and transgenic seedlings were slightly wilted. Seedling growth was most affected by elevated temperature. After $7 \mathrm{~d}$ of heat exposure, both WT and transgenic lines had pale green cotyledons. Only $17 \%$ (i.e. 4/24) of the WT plants survived exposure to elevated temperature, while $92 \%$ (44 out of 48) of the transgenic plants (both L1 and L2) survived exposure to elevated temperature.

\section{Improved root branching in EsDREB2B transgenic plants in response to stress}

DREB2 proteins such as CAP2 and PgDREB2B have been found to enhanced stress tolerance through regulating root architecture (i.e. root length and lateral root number) [21,43]. We analyzed the root length and lateral root number (Figure 6B, C), and leaf number (Additional file 5: Figure $\mathrm{S}$ ) of WT and transgenic plants overexpressing EsDREB2B under stress Under non-stress (NS) condition, there were no differences between WT and transgenic plants regarding root length and root number (Figure 6B, C). Root length in L1 transgenic plants was 


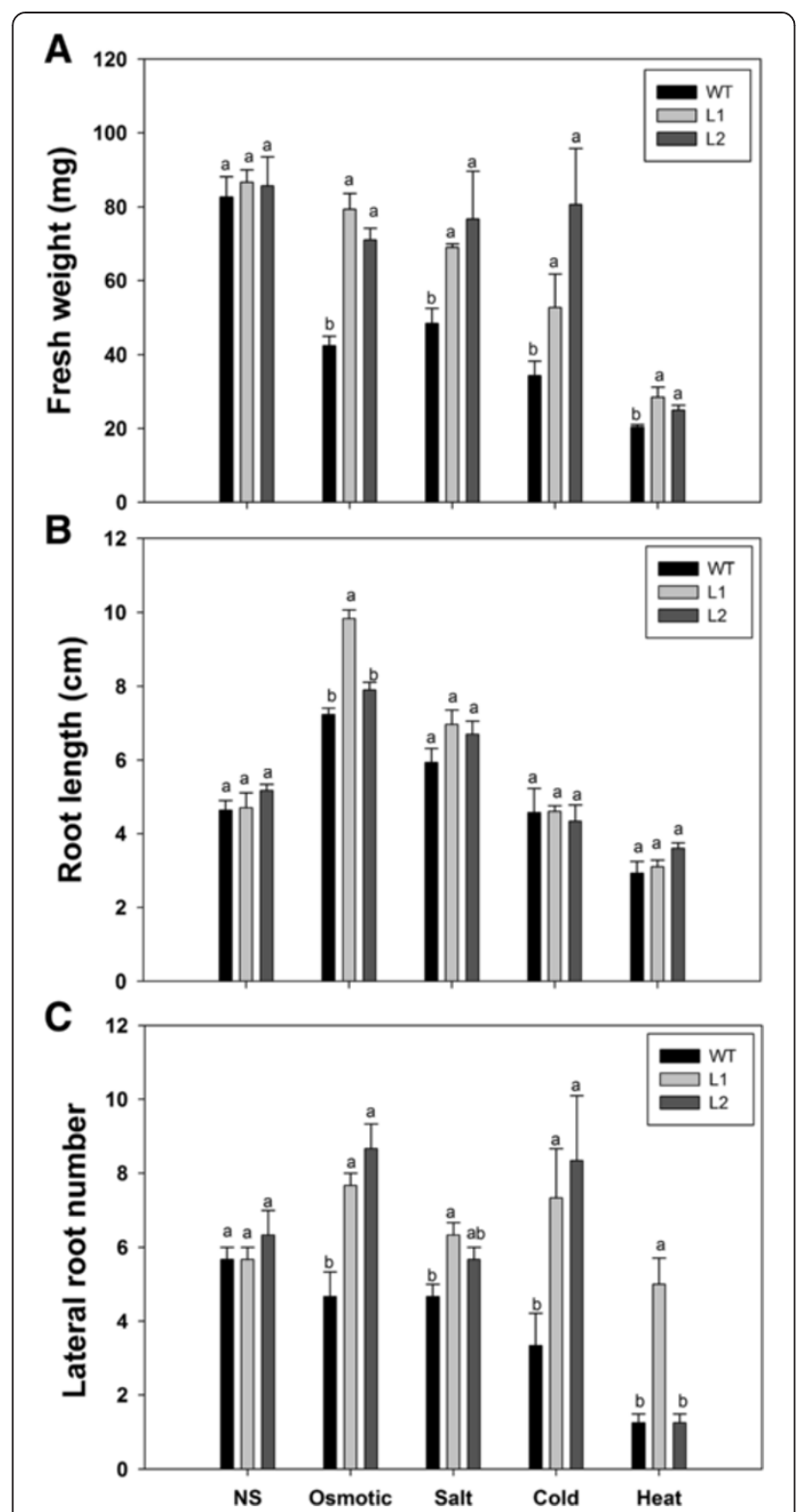

Figure 6 Fresh weight and root architecture comparison of non-transformed (WT) plants and two EsDREB2B transgenic tobacco lines under osmotic, salt, cold and heat stresses. For osmotic and salt stresses, the two-week old seedlings grown on MS medium transplanted to MS medium supplemented with $200 \mathrm{mM}$ mannitol and $200 \mathrm{mM} \mathrm{NaCl}$ for $20 \mathrm{~d}$. For cold and heat stresses, the two-week-old seedlings grown in MS medium were treated for $20 \mathrm{~d}$ at $4^{\circ} \mathrm{C}$ or $7 \mathrm{~d}$ at $42^{\circ} \mathrm{C}$, respectively. For non-stress control (NS), seedlings were grown in MS culture and kept in normal growth conditions (at $28^{\circ} \mathrm{C}$ ). All plants were grown on vertical MS Plates. A fresh weight (expressed in $\mathrm{mg}$ ), B root length (expressed in $\mathrm{cm}$ ), and $\mathbf{C}$ lateral root number were determined after treatments. Results are presented means \pm SE $(n=24$ seedlings). $\mathbf{a}-\mathbf{b}$ : there are no significant differences $(p \geq 0.05)$ between the lines with the same characters, and significant differences $(p<0.05)$ between lines with different characters. Significant difference comparison was carried out within stress. significantly higher in response to osmotic-stress (Figure 6B). With the exception of L2 exposed to elevated temperature, all transgenic plants had significant more lateral roots in response to each of the four treatments (Figure 6C), while leaf number was unchanged (Additional file 5: Figure S5). These results indicated that overexpression of EsDREB2B can enhance tolerance to osmotic-, salt- and temperature-stress.

\section{EsDREB2B transgenic plants were less damaged and} accumulated higher levels of proline under stress

We monitored several plant stress responses at the physiological level (Figure 7). The MDA, proline and chlorophyll contents did not significantly differ among the WT, L1 and L2 plant lines after $20 \mathrm{~d}$ of non-stress (NS) growth (Figure 7A, B, C). In response to all four treatments, L1 transgenic plants had significantly less damage as measured by MDA content when compared with WT plants (Figure 7A). With the exception of salinitystress, L2 transgenic plants had the same response as L1 transgenic plants. Transgenic plants also accumulated significantly more proline as compared to WT lines under all the stress conditions (Figure 7B). These results indicated that overexpression of EsDREB2B increases the amount of proline in response to osmotic-, salt- and temperaturestress. For chlorophyll content, significant differences between transgenic and WT plants were observed only in response to elevated temperature (Figure $7 \mathrm{C}$ ).

\section{Discussion}

DRE-binding (DREB) proteins are key components of abiotic stress tolerance in plants $[7,8]$. DREB2-type transcription factors respond to an array of stressors (i.e. dehydration, salinity, cold, heat and exogenous ABA) and are documented to induce the expression of numerous, downstream, abiotic-stress related genes. Due to their importance, DREB2 genes have been identified in a large number of plant species including soybean [35], rice [38], corn [40], barley [23], chickpea [21] and Arabidopsis [24]. We hypothesize that the identification and characterization of DREB2B homologues from plants adapted dry environments, such as E. songoricum, will provide unique insights to DREB function and gene control.

With few notable exceptions (such as GmDREB2B), DREB2 genes are induced by both dehydration and heat shock $[7,8]$. Steady state transcript amounts of EsDREB2B do not respond to environmental factors as a typical DREB2B gene. EsDREB2B steady state transcript amounts are induced in response to all experimental treatments used in this study: osmotic stress, salinity, low temperature, exogenous ABA, elevated temperature, oxidative stress, heavy metal and wounding. Strong induction in response to dehydration, salinity and elevated temperature coupled with a 


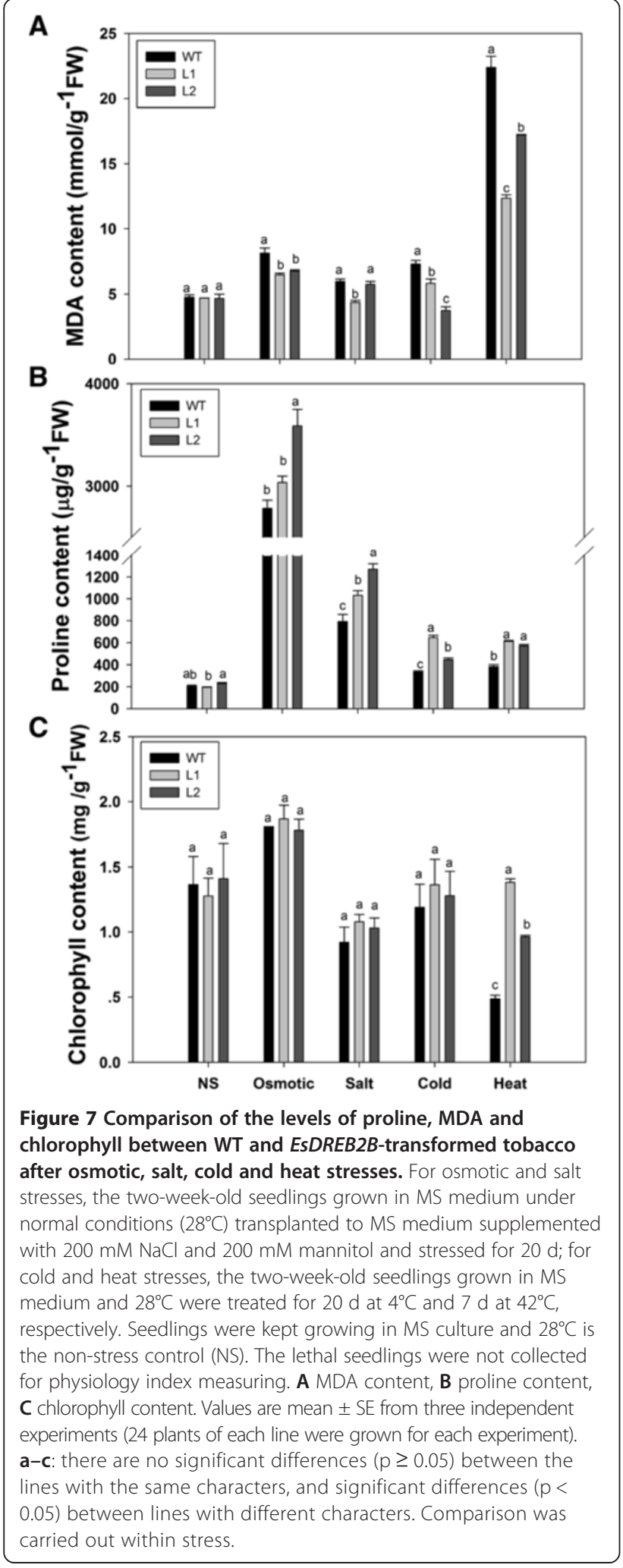

lack of induction by low temperature is characteristic of AtDREB2A, AtDREB2B, OsDREB2A and CAP2 [21,24,38]. EsDREB2B, like GmDREB2A [22], ZmDREB2A [40] and
OsDREB2B [38], exhibited strong induction in response to dehydration, salinity, elevated temperature and low temperature. Interestingly, steady state transcript amounts of the legume-specific, truncated sequences EsDREB2B, $C A P 2$ and $G m D R E B 2 B / C$ are induced by exogenous ABA treatment $[21,35]$.

The EsDREB2B deduced polypeptide sequence is a member of the AP2/ERF protein family and most similar to deduced polypeptide sequences from chickpea (CAP2) [21] and soybean (GmDREB2B) [35]. The CMIV-1, CMIV-2 and AP2 domains are found in all previously characterized DREB2 (subtype 1) deduced polypeptides suggesting conserved function of these domains. CMIV-1 and CMIV-2 have been characterized as NLS motifs and AP2 has been determined to act as a DNA-binding domain [24,34,39]. The most intensely studied DREB2 genes (i.e. AtDREB2A/ 2C, OsDREB2B and ZmDREB2A) contain a CMIV-3 domain in the carboxy-terminal region and the domain has been demonstrated to function as a strong transactivation domain [38-40]. The legume-specific, truncated DREB2 group (i.e. CAP2 and EsDREB2B) contains the CMIV-4 domain. In CAP2, the CMIV-4 domain has been shown to be required for heat tolerance in yeast and may play a critical role in the transactivation of these truncated proteins $[21,42]$. Shorter DREB2 deduced polypeptides, such as AtDREB2E, AtDREB2H and OsDREB2A lack both the CMIV-3 and CMIV-4 domains and are reported to either be non-responsive to stress or respond to stress without transactivation activity [36,38].

Based upon shared motifs and structural features, we have classified DREB2-type (subtype 1) deduced polypeptides proteins into three groups: 1) non-truncated deduced polypeptides that possess a CMIV-3 domain, 2) deduced polypeptides that lack both the CMIV-3 and CMIV-4 domains and 3) truncated (legume-specific) deduced polypeptides that share a unique CMIV-4 motif. Group 1 DREB2 genes are reported to be significant stress response genes $[8,24,36,39]$ while Group 2 DREB2 genes are not considered to play a key role in stress response $[36,38]$. These results suggest the possibility that the different groups of DREB2-type proteins with group-specific motifs may have different transactivation mechanisms, and may play different roles in plant stress response.

The ability of DREB2 homologues to enhance osmotic stress tolerance in transgenic plants has been extensively studied $[7,8]$. Overexpression of AtDREB2A-CA in transgenic Arabidopsis results in greatly enhanced drought- and heat-stress tolerance, slightly enhanced freezing tolerance and no salt-tolerance $[39,44]$. Similar results have been observed with transgenic Arabidopsis overexpressing OsDREB2B;2 [38], ZmDREB2A [40] and GmDREB2A [22]. Overexpression of CAP2 in transgenic tobacco resulted in enhanced osmotic, salt- and heat-tolerance 
$[21,42]$; with the exception of heat-stress tolerance, similar results have been observed with transgenic tobacco overexpressing PgDREB2A [43] and LcDREB [11]. Overexpression of EsDREB2B in transgenic tobacco was demonstrated to enhance osmotic- and heat-stress tolerance and also enhance cold- and salt-stress tolerance.

Unique among DREB2-type homologues, only EsDREB2B and $O s D R E B 2 B$ transcript amounts are strongly induced by dehydration, salt, low temperature, elevated temperature and exogenous ABA. However, transgenic Arabidopsis overexpressing OsDREB2B have enhanced tolerance to drought- and heat-stress only. The legume-specific, truncated EsDREB2B transcript is unique among DREB2 homologues in the breadth of transcriptional response and overexpression of the sequence in transgenic tobacco provides enhanced tolerance to osmotic-, salt-, cold- and heatstress. Further experiments are needed to characterize the mechanisms controlling the accumulation of EsDREB2B transcript and to establish the downstream target promoters potentially regulated by this protein in response to a suite of abiotic stresses.

In DREB2B overexpressing transgenic plants (i.e. Arabidopsis and rice), a common observation is reduced foliar growth as compared to control plants [22,38-40]. In sharp contrast, EsDREB2B overexpressing transgenic tobacco demonstrated enhanced growth relative to non-transformed controls under stress conditions. EsDREB2B transgenic plants also exhibited no morphological differences as compared to the nontransformed controls. Root architecture and morphology is an important feature of plant growth and development [45]. EsDREB2B transgenic tobacco plants had significantly increased numbers of lateral roots. It has been hypothesized that increasing primary root length (e.g. mining for water) and/or increasing lateral root number (e.g. scavenging for water) represent important adaptations to water-limiting environments [46].

The ability to modify essential metabolic processes is key to adapting to adverse environmental conditions. The accumulation of proline is a common response to both biotic and abiotic stress in plants [47] and is postulated to be an adaptive response to declining water potential [48]. Transgenic Arabidopsis and rice plants overexpressing DREB1/CBF have been shown to accumulate proline under control conditions [27]. Several subsequent studies have demonstrated that transgenic plants overexpressing DREB genes will accumulate proline under control conditions and in response to salt-stress [49], osmotic-stress [50], and CuSO4stress [11]. Overexpression of EsDREB2B can influence the physiological and biochemical responses of transgenic tobacco and mediate the accumulation of proline in response to osmotic-, salt-, cold- and heat-stress.

\section{Conclusions}

The cDNA EsDREB2B was isolated from the desert plant E. songoricum. EsDREB2B encodes a truncated DREB2 deduced polypeptide is a novel truncated DREB2-type protein apparently unique to the Fabaceae. In E. songoricum, EsDREB2B steady state transcript was detectable under non-stress conditions and accumulated in response to a variety of abiotic stresses. EsDREB2B was targeted to the nucleus and exhibited transactivation activity of a GAL4-containing reporter gene. EsDREB2B was shown to be a functional DREB protein able to: 1 ) impart abiotic stress tolerance in transgenic yeast and 2) impart abiotic stress tolerance in transgenic tobacco. In our study, the heterologous expression of EsDREB2B enhanced the tolerance of transgenic tobacco to osmotic-, salt-, cold- and heat-stress. We conclude that EsDREB2B is a promising candidate gene for the development of crops with multiple stress tolerances.

\section{Methods}

\section{Plant material and stress treatments}

Eremosparton songoricum (Litv) Vass. seeds were collected from the Gurbantunggut Desert, in the Xinjiang Uygur autonomous region of P. R. China $\left(88^{\circ} 24^{\prime} 67^{\prime \prime}\right.$, $45^{\circ} 58^{\prime} 11^{\prime \prime} \mathrm{N}, 667 \mathrm{~m}$ asl) [32]. Seeds were soaked in $98 \%$ $(\mathrm{v} / \mathrm{v})$ sulfuric acid for 10-15 minutes to break the physical dormancy of the seeds, washed with $\mathrm{ddH}_{2} \mathrm{O}$ and sown onto moist filter paper. Seedlings were grown in covered petri plates under controlled conditions $\left(25^{\circ} \mathrm{C}\right.$, $12 \mathrm{~h}$ darkness, $100 \mathrm{~mol} \mathrm{~m}^{-2} \mathrm{~s}^{-1}, 60 \% \mathrm{RH}$ ) prior to stress treatment. Plants were monitored daily. The filter paper was never allowed to dry and the plants were not submerged.

E. songoricum seedlings were exposed to one of eight stress treatments including PEG6000, salinity, low temperature, elevated temperature, exogenous ABA, $\mathrm{H}_{2} \mathrm{O}_{2}$, exposure to heavy metal and wounding. Twoweek-old seedlings were transferred to new petri plates and the filter paper was saturated with $8 \mathrm{~mL}$ of one of the following solutions: $20 \%(\mathrm{w} / \mathrm{v})$ PEG6000, $250 \mathrm{mM}$ $\mathrm{NaCl}, 100 \mu \mathrm{M} \mathrm{ABA}, 50 \mathrm{mM} \mathrm{H} \mathrm{O}_{2}$, or $0.5 \mathrm{mM} \mathrm{CuSO}_{4}$. For the cold and heat treatments, two-week-old seedlings were incubated at $4^{\circ} \mathrm{C}$ or $42^{\circ} \mathrm{C}$, respectively. For mechanical wounding treatment, leaves were cut six times per leaf with a blade [51]. For each time point (i.e. $0.5 \mathrm{~h}, 2 \mathrm{~h}, 6 \mathrm{~h}, 12 \mathrm{~h}$, and $24 \mathrm{~h}$ ), 3-4 entire plants were harvested, pooled and flash frozen in liquid nitrogen. To serve as a control, two-week-old seedlings were transferred to new petri plates and the filter paper was saturated with $8 \mathrm{~mL}$ of water. All plants were maintained under identical growth conditions (i.e. light and $\mathrm{RH}$ ). To obtain EsDREB2B, two-week-old E. songoricum seedlings were exposed to osmotic stress (i.e. 20\% (w/v) polyethylene glycol (PEG6000)) for 
$12 \mathrm{~h}$. Harvested samples were flash frozen in liquid nitrogen and stored at $-80^{\circ} \mathrm{C}$ prior to RNA extraction.

\section{RNA extraction and CDNA synthesis}

Total RNA extraction was performed using the RNAiso ${ }^{\text {tw }}$ plus reagent (Takara, Japan) following the manufacturer's instructions with little modification. Genomic DNA contamination was eliminated using RNase-free DNase I (Takara, Japan). The RNA concentration, purity, and integrity were determined using a NanoDrop ND-2000 spectrophotometer (Thermo Fisher Scientific, USA) and were visually assessed via gel electrophoresis $(1.2 \%(\mathrm{w} / \mathrm{v})$ agarose gel). Only RNA samples with a $260 / 280$ ratio of between 1.9 and 2.1 and a 260/230 ratio of higher than 2.0 were used for the subsequent analyses. First-strand cDNA were synthesized from $1 \mu \mathrm{g}$ of total RNA, $1 \mu \mathrm{l}$ of oligo-dT, $1 \mu \mathrm{l}$ of random six-mers and $4 \mu \mathrm{l}$ of $5 \times$ Primerscript Buffer (Takara, Japan). The RT-PCR reaction was carried out at $37^{\circ} \mathrm{C}$ for $30 \mathrm{~min}$ on a $\mathrm{C} 1000^{\text {rm }}$ Thermal Cycler (Bio-Rad, USA) at a final volume of $20 \mu \mathrm{l}$, and inactivation of the enzyme was completed at $85^{\circ} \mathrm{C}$ for 5 min. All of the cDNA were stored at $-20^{\circ} \mathrm{C}$.

\section{Cloning the EsDREB2B CDNA}

A conserved partial sequence (ca $300 \mathrm{bp}$ ) of the EsDREB2B cDNA was amplified from $E$. songoricum cDNA using degenerate primers based upon the AP2 DNA-binding domain of DREB2 deduced polypeptides (G-f: 5'TGAAA CACTGGCAAAATGGAWNGARTAYA-3' and G-r:5'CCATACATAGCCATAGCAGCTTCRTCRTANGC-3') [52]. A full-length EsDREB2B cDNA was amplified from $E$. songoricum $\mathrm{CDNA}$ using primers derived from the $5^{\prime}$ and 3'-UTR sequences of DREB2 from Cicer arietinum (DQ321719) and soybean (AAT1242323) (D-f: 5' '-TTCTTCCTCAAATGCCTTCTGG-3' and D-r: 5' -AT GCAA AACTTACATCAAGACAATG-3'). All primers were designed using Primer Premier 5.0.

\section{Cloning of a partial EsDREB2B gene}

DNA was isolated from whole plants of E. songoricum using a C-TAB extraction method [53]. To check for the existence of introns within the EsDREB2B gene, an ORF-based PCR amplification (primers ORF-f: $5^{\prime}$-ATG AGTGCAACTTGCATGCA-3' and ORF-r: 5'-AGACAATGAAGGATCGGAGG-3') was performed using both DNA and cDNA as templates. PCR was performed using Ex Taq ${ }^{\mathrm{Tm}}$ (TaKaRa, Japan), and the reaction conditions were as follows: 30 cycles of denaturation at $94^{\circ} \mathrm{C}$ for $30 \mathrm{~s}$, annealing at $55^{\circ} \mathrm{C}$ for $30 \mathrm{~s}$, and extension at $72^{\circ} \mathrm{C}$ for $45 \mathrm{~s}$. An initial denaturation step of $5 \mathrm{~min}$ at $95^{\circ} \mathrm{C}$ and a final elongation step at $72^{\circ} \mathrm{C}$ for $10 \mathrm{~min}$ were also performed. The final amplification products were checked on a $1.2 \%(\mathrm{w} / \mathrm{v})$ agarose gel. Amplicons were purified using the EZ Spin Column DNA Gel Extraction
Kit (BIO BASIC INC, Canada) and ligated into the $\mathrm{pMD}^{\mathrm{mw}} 19-\mathrm{T}$ vector (according to the manufacturer's instructions). Positive colonies containing the recombinant plasmids were then sent to the Beijing Genomics Institute (Beijing, China) for DNA sequencing.

\section{Phylogenetic analysis and conserved protein motif prediction}

The resulting DNA sequence and the deduced polypeptide sequences, along with the BLAST algorithm, were used to query the appropriate databases in the National Center for Biotechnology Information (NCBI). Multiple sequence alignment of 31 deduced DREB amino acid sequences was performed using Clustal-W in conjunction with MEGA 5.1 [54]. Phylogenetic trees were generated by the neighbor-joining (NJ) method using MEGA 5.1 (poisson correction and pairwise deletion). Support for nodes on the estimated phylogeny was tested with 1000 bootstrap replicates using the neighbor-joining method [55]. Motif detection was performed with the online tool MEME [56] using the parameters: 0 or 1 occurrence of a single motif per sequence, motif width ranges of 6 to 50 amino acids, and 10 as the maximum number of motif. Motif alignment was performed using MAST (motif alignment and search tool) using default parameters [57].

\section{Gene expression analysis by qRT-PCR in response to abiotic stress}

RNA was isolated from two-week old seedlings exposed to one of eight stress treatments at the time points indicated (see above). To test differential tissue expression, samples of the roots, stems and leaves from two- weekold seedlings grown in normal conditions were collected separately. All cDNA samples were diluted 5-fold with RNase-free water before being used as the template for quantity analysis. QRT-PCR primers were designed outside of the AP2 domain (Dq-f: 5' -AGCCGAGAAGCCTGTTA3' and Dq-r: 5' - CCCAAGTCCTTTGCCTA -3). QRTPCR reactions were performed using SYBR Premix Ex Taq $^{T M}$ (Takara, Japan) and the CFX96 Real-Time PCR Detection System (Bio-Rad, USA). The reaction mixture consisted of $2 \mu \mathrm{l}$ of the diluted cDNA samples, $0.4 \mu \mathrm{l}$ each of the forward and reverse primers $(10 \mu \mathrm{M}), 10 \mu \mathrm{l}$ of the real-time master mix and $7.2 \mu \mathrm{l}$ of the PCR-grade water for a final volume of $20 \mu \mathrm{l}$. Two biology replicates were performed for all of the samples, and three technical replicates of each of the biological replicates with a no-template control (NTC) were made. QRT-PCR protocol was as follows: $30 \mathrm{~s}$ of initial denaturation at $95^{\circ} \mathrm{C}, 40$ cycles at $94^{\circ} \mathrm{C}$ for $5 \mathrm{~s}$, and annealing at $60^{\circ} \mathrm{C}$ for $30 \mathrm{~s}$. The relative expression levels of the $E s D R E B 2 B$ gene were calculated relative to the control (at $0 \mathrm{~h}$ ). Selection of E. songoricum reference genes is based upon our previous research [32]. The combination of $E s E F$ and $E s \alpha-T U B$ are sufficient to reliably normalize 
the qRT-PCR data in various abiotic stress conditions, and $E s G A P D H$ and $E s \beta-T U B 2$ are suitable for different tissue samples.

\section{Subcellular localization analysis}

To determine the subcellular localization of the gene products, the full-length ORF of ESDREB2B (without the termination codon) was amplified using the specific primers sub-f and sub-r (sub-f: 5' -ATGAGTGCAACTTGCATGC3' and sub-r: 5'-AGACAATGAAGGATCGGAGG-3'). The ORF was fused to the $5^{\prime}$ end of the GFP coding sequence and sub-cloned to pBI221 under control of the CaMV 35S promoter. The fusion construct (p35SEsDREB2B: GFP) and the control (p35S-GFP) were transformed into onion epidermis cells by particle bombardment (Bio-Rad, Hercule, CA). Green fluorescence was observed under a confocal microscope (Olympus FV500, Olympus, Japan).

\section{Trans-activation assay using yeast one-hybrid system}

The entire ORF of EsDREB2B was amplified with specific primers (Y-f and Y-r), and the primer sequences were $\mathrm{Y}-\mathrm{f}$ : 5'-GTCGACTGATGAGTGCAACTTGCATGC-3' and Yr: 5'-CTGCAGTCAAGACAATGAAGGATCGG-3'). The amplification product was inserted between the PstI and SalI restriction sites of the yeast expression vector pBridge, which also contained the binding domain (BD) of GAL4 (pBridge -BD-EsDREB2B). The recombinant plasmids were introduced into Saccharomyces cerevisiae (strain AH109 yeast strain carrying the His3 and LacZ reporter genes) per the manufacturer's instructions (Clontech, USA). Yeast cells containing only GAL4 (pBridge-BD-GAL4) or the pBridge vector (pBridge- $\mathrm{BD}$ ) were used as positive and negative controls, respectively. Successful transformants were selected by growth on SD media without His and Trp at $30^{\circ} \mathrm{C}$ for 3 days. Positive colonies were confirmed by yeast colony PCR. In addition, the $\beta$-galactosidase activity of the transformant colonies was examined by incubating the colonies in $\mathrm{Z}$ buffer with $\mathrm{x}$-gal at $30^{\circ} \mathrm{C}$.

\section{EsDREB2B stress tolerance studies in yeast}

The ORF of EsDREB2B was amplified from pMD19-TEsDREB2B using the primer pair YE-f and YE-r and contained the sequences for the BamHI and $K p n I$ restriction enzymes sites. The primer sequences were: YE-f: 5' -GGTACCATGAGTGCAACTTGCATGCA-3' and YE-r: 5'-CGGATCCGTCAAGACAATGAAGGAT CGG-3'. The ORF was inserted into the BamHI and $K p n I$ sites of the yeast expression vector pYES2 (Invitrogen, USA), which contains a URA3 selection marker driven by the GAL1 promoter. Subsequently, the pYES2-EsDREB2B plasmid and the empty pYES2 control plasmids were introduced into yeast strain INVSc1 (Invitrogen, USA) using a lithium acetate procedure, according to the pYES2 vector kit instructions (Invitrogen, USA). The transformants were screened by growth on a uracil-deficient synthetic complete (SC-ura) medium with $2 \%(\mathrm{w} / \mathrm{v})$ glucose at $30^{\circ} \mathrm{C}$. Positive colonies were verified by plasmid PCR and double digestion (with $\mathrm{BamHI}$ and $\mathrm{Kpn}$ I) analysis.

To investigate the expression of EsDREB2B in the transgenic yeast, total RNA from yeast cells harboring pYES2-EsDREB2B were isolated using the TRIzol Reagent (Invitrogen, USA). A semi-quantitative RT-PCR assay was performed using the YE-f and YE-r primer pair for the target gene, with ACT1 as the reference gene; the ACT1 primer set conforms to that of Teste et al. [58]. For the stress assay, yeast cells harboring both pYES2-EsDREB2B and the empty pYES2 vector (control) were incubated in SC-ura liquid medium containing $2 \%$ $(\mathrm{w} / \mathrm{v})$ glucose at $30^{\circ} \mathrm{C}$ for approximately $20 \mathrm{~h}$. The densities of the yeast cell cultures were measured (by OD600) after incubation. The culture samples were adjusted to contain an equal number of cells $(\mathrm{OD} 600=0.4)$ in $10 \mathrm{ml}$ of induction medium (SC-ura medium supplemented with $2 \% \mathrm{w} / \mathrm{v}$ galactose) and cultivated for $36 \mathrm{~h}$ to promote the expression of the EsDREB2B gene. After incubation, the yeast cell densities were recalculated and again adjusted to contain an equal number of cells $(\mathrm{OD} 600=1)$ in $200 \mu \mathrm{l}$ solutions with different chemicals for the abotic stress assays. For the drought and salt stresses, the same quantity of culture sample was resuspended in $30 \%(\mathrm{w} / \mathrm{v})$ PEG6000 or $5 \mathrm{M} \mathrm{NaCl}$, respectively, at $30^{\circ} \mathrm{C}$ for $36 \mathrm{~h}$. For the cold and heat stresses, yeast cells were resuspended in SC-ura medium and either incubated in a $-20^{\circ} \mathrm{C}$ alcohol bath for $24 \mathrm{~h}$ or in a water bath at $53^{\circ} \mathrm{C}$ for $24 \mathrm{~h}$ [59]. For oxidative stress, yeast cells were incubated in $20 \mathrm{mM} \mathrm{H}_{2} \mathrm{O}_{2}$ at $30^{\circ} \mathrm{C}$ for $8 \mathrm{~h}$. For the control, an equivalent number of yeast cells was resuspended in $200 \mu \mathrm{l}$ of sterile water and incubated at $30^{\circ} \mathrm{C}$ for $36 \mathrm{~h}$. After the stress treatments, the cells were 10 -fold serially diluted $\left(1,10^{-1}, 10^{-2}, 10^{-3}\right.$, and $\left.10^{-4}\right)$, and $2 \mu \mathrm{l}$ aliquots of each dilution were spread on SC-ura medium (supplemented with $2 \% \mathrm{w} / \mathrm{v}$ glucose) and incubated at $30^{\circ} \mathrm{C}$ for 3 days to observe their growth performance. Each experiment was carried out in triplicate.

\section{The generation of transgenic tobacco plants}

To verify the stress tolerance function of the EsDREB2B CDNA, complete ORF was inserted in the plant expression vector pCAMBIA 1301, which was digested by PstI and $K p n I$. The pCAMBIA 1301-EsDREB2B vector was introduced into tobacco (Petite Havana SR1) by Agrobacterium tumefaciens-mediated leaf dish transformation (LBA4404) [60]. The transgenic nature of the tobacco was confirmed by a hygromycin screen $(50 \mu \mathrm{g} / \mathrm{ml})$ as well as PCR and RT-PCR amplification using the EsDREB2B gene specific primer set ORF-f and ORF-r (see above). 


\section{Evaluation of transgenic tobacco plants for abiotic stress tolerance}

Two- week-old homozygous T2 transgenic tobacco seedlings were used in various stress tolerance experiments. The WT line and two randomly selected transgenic lines designated L1 and L2 were used for further analysis. Seeds were sown onto solid MS medium plates and allowed to germinate and grow in a $28^{\circ} \mathrm{C} / 25^{\circ} \mathrm{C}$ growth chamber under $8 \mathrm{~h}$ darkness and $60 \% \mathrm{RH}$. For subsequent treatment all seedlings (including controls) were transplanted to solid MS medium. For osmotic stress and salinity, two-week-old seedlings were transplanted to solid MS medium supplemented with either $200 \mathrm{mM}$ mannitol or $200 \mathrm{mM} \mathrm{NaCl}$ for $20 \mathrm{~d}$ and grown vertically. For the low temperature and elevated temperature, two-week-old seedlings were transplanted to solid MS medium maintained for $20 \mathrm{~d}$ at $4^{\circ} \mathrm{C}$ or $7 \mathrm{~d}$ at $42^{\circ} \mathrm{C}$, respectively and grown vertically. For a control, seedlings were grown vertically in MS culture and kept in normal growth conditions (at $28^{\circ} \mathrm{C}$ ). After the treatment, plant growth parameters such as root length, lateral root number, leaf number, and fresh weight were monitored. The length of the main root was measured with a ruler. Physiological indexes, including the amounts of proline, methane dicarboxylic aldehyde (MDA) and chlorophyll, were measured after the stress treatments according to the methods described [61-63]. The plant's phenotype was photographed before and after the stress treatment.

\section{Statistical analysis}

Statistical analyses were performed using SPSS software (standard version 11.5 released for Windows, SPSS Inc., IL, USA). All data were analyzed using a one-way analysis of variance (ANOVA) at the 95\% confidence level. An LSD multiple comparison test was then used to compare the significant differences. The data shown are the mean values \pm SE of at least three replicates, and the significance level relative to controls is $\mathrm{P}<0.05$. The figures were generated using Sigmaplot10.0. The imageprocessing software used was Adobe Illustrator CS.

\section{Additional files}

Additional file 1: Figure S1. Sequence logo for the CMIV-4 motif of

the truncated proteins.

Additional file 2: Figure S2. Phylogenetic tree of EsDREB2B and homolog proteins. E. songoricum (EsDREB2B, pink and bold), soybean (red), Arabidopsis (blue), rice (cyan) and other proteins with high sequence identities with EsDREB2B. The neighbor-joining tree is based on the Jone-Taylor-Thornton model (pairwise deletion) with an alignment of the complete protein sequence. Bootstrap values from 1000 replicates were used to assess the robustness of the tree. Bootstrap values $>50$ are shown.

Additional file 3: Figure S3. Tissue expression pattern of ESDREB2B in two-week old E. songoricum seedling under normal condition. The relative gene expression level of ESDREB2B gene were calculated relative to leaf sample using $2^{-\Delta \Delta C T}$ method. Error bars indicate SD $(n=3)$.

Additional file 4: Figure S4. Semi-quantitative RT-PCR analysis of induced gene expression pattern of ESDREB2B in recombinant yeast. The suppressive and galactose-induced expression of control yeast (pYES2) for $36 \mathrm{~h}$ (Lanes 1, 2). The suppressive expression of recombinant yeast (pYES2-EsDREB2B) for $36 \mathrm{~h}$ (lane3) and galactose-induced expression of recombinant yeast for $12 \mathrm{~h}$ (lane 4), $24 \mathrm{~h}$ (lane 5), $36 \mathrm{~h}$ (lane 6), $48 \mathrm{~h}$ (lane 7) and $60 \mathrm{~h}$ (lane 8).

Additional file 5: Figure S5. Phenotype and leaf number comparison of non-transformed (WT) plants and two ESDREB2B transgenic tobacco lines under osmotic, salt, cold and heat stresses. A The photograph was taken beforeand after stress; B leaf number were counted after stress treatments. Results are presented means \pm SE ( $n=24$ seedlings). Significant difference comparison was carried out within stress.

\section{Abbreviations}

TF: Transcription factors; DREB: Dehydration-responsive element binding protein; ORF: Open reading frame; NJ: Neighbor-joining; PEG: Polyethylene glycol; qRT-PCR: Quantitative RT-PCR; NTC: No-template control; ABA: Exogenous abscisic acid; NLS: Nuclear localization sequence; NRD: negative regulatory domain; MDA: Methane dicarboxylic aldehyde.

\section{Competing interests}

The authors declare that they have no competing interests.

\section{Authors' contribution}

XSL performed the gene cloning, sub-cellular location and transgenic tobacco assays, and wrote the manuscript. DYZ designed, supervised and financed this work, and assisting with editing the manuscript. HYL carried out the gene expression and transgenic yeast experiments. YCW provided advice on the yeast activation assay, transgenic yeast assay and assisted with data analysis. YMZ designed and prepared the manuscript. AJW assisted with data analysis and edited the manuscript. All authors read and approved the final manuscript.

\section{Acknowledgements}

This research was supported by the National Basic Research Program of China (2014CB954203) and the National Natural Science Foundation (U1170304). The support provided by the Chinese Scholarship Council (Co-education PhD award to XL, File No 201204910321) is acknowledged.

\section{Author details}

${ }^{1}$ Key Laboratory of Biogeography and Bioresource in Arid Land, Xinjiang Institute of Ecology and Geography, Chinese Academy of Sciences, Xinjiang Urumqi 830011, China. ${ }^{2}$ University of Chinese Academy of Sciences, Beijing 100049, China. ${ }^{3}$ Department of Plant Biology, Southern Illinois University, Carbondale, IL 62901-6899, USA.

Received: 13 September 2013 Accepted: 5 February 2014

Published: 10 February 2014

\section{References}

1. Cramer GR, Urano K, Delrot S, Pezzotti M, Shinozaki K: Effects of abiotic stress on plants: a systems biology perspective. BMC Plant Biol 2011, 11:163.

2. Jenks MJ, Wood AJ: Genes for Plant Abiotic Stress. Ames: Wiley-Blackwell Press; 2010.

3. Boyer JS: Plant productivity and environment. Science 1982, 218:443-448.

4. Valliyodan B, Nguyen HT: Understanding regulatory networks and engineering for enhanced drought tolerance in plants. Curr Opin Biotech 2006, 9:189-195.

5. Wang W, Vinocur B, Altman A: Plant responses to drought, salinity and extreme temperatures: towards genetic engineering for stress tolerance. Planta 2003, 218:1-14.

6. Hussain SS, Kayani MA, Amjad M: Transcription factors as tools to engineer enhanced drought stress tolerance in plants. Biotechnol Progr 2011, 27:297-306. 
7. Nakashima K, Ito Y, Yamaguchi-Shinozaki K: Transcriptional regulatory networks in response to abiotic stresses in Arabidopsis and grasses. Plant Physiol 2009, 149:88-95.

8. Lata C, Prasad M: Role of DREBs in regulation of abiotic stress responses in plants. J Exp Bot 2011, 62:4731-4748.

9. Xu ZS, Chen M, Li LC, Ma YZ: Functions and application of the AP2/ERF transcription factor family in crop improvement. J Integr Plant Biol 2011, 53:570-585.

10. Agarwal P, Agarwal PK, Nair S, Sopory SK, Reddy MK: Stress-inducible DREB2A transcription factor from Pennisetum glaucum is a phosphoprotein and its phosphorylation negatively regulates its DNA-binding activity. Mol Genet Genomics 2007, 277:189-198.

11. Ban Q, Liu G, Wang Y: A DREB gene from Limonium bicolor mediates molecular and physiological responses to copper stress in transgenic tobacco. J Plant Physiol 2011, 168:449-458.

12. Xianjun $P$, Xingyong $M$, Weihong $F$, Man $S$, Liqin C, Alam I, Lee BH, Dongmei Q, Shihua S, Gongshe L: Improved drought and salt tolerance of arabidopsis thaliana by transgenic expression of a novel DREB gene from Leymus chinensis. Plant Cell Rep 2011, 30:1493-1502.

13. Xiong Y, Fei SZ: Functional and phylogenetic analysis of a DREB/CBF-like gene in perennial ryegrass (Lolium perenne L.). Planta 2006, 224:878-888

14. Mizuno S, Hirasawa Y, Sonoda M, Nakagawa H, Sato T: Isolation and characterization of three DREB/ERF-type transcription factors from melon (Cucumis melo). Plant Sci 2006, 170:1156-1163.

15. Tillett RL, Wheatley MD, Tattersall EAR, Schlauch KA, Cramer GR, Cushman JC: The vitis vinifera $\mathrm{C}$-repeat binding protein 4 (VVCBF4) transcriptional factor enhances freezing tolerance in wine grape. Plant Biotechnol J 2012, 10:105-124.

16. Hong JP, Kim WT: Isolation and functional characterization of the Ca-DREBLP1 gene encoding a dehydration-responsive element bindingfactor-like protein 1 in hot pepper (Capsicum annuum L. cv. Pukang). Planta 2005, 220:875-888.

17. Zhang X, Fowler SG, Cheng HM, Lou YG, Rhee SY, Stockinger EJ, Thomashow MF: Freezing-sensitive tomato has a functional CBF cold response pathway, but a CBF regulon that differs from that of freezing-tolerant Arabidopsis. Plant J 2004, 39:905-919.

18. Chen JQ, Meng XP, Zhang Y, Xia M, Wang XP: Over-expression of OsDREB genes lead to enhanced drought tolerance in rice. Biotechnol Lett 2008, 30:2191-2198.

19. Chen M, Wang QY, Cheng XG, Xu ZS, Li LC, Ye XG, Xia LQ, Ma YZ: GmDREB2, a soybean DRE-binding transcription factor, conferred drought and high-salt tolerance in transgenic plants. Biochem Bioph Res Co 2007, 353:299-305

20. Huang B, Jin L, Liu JY: Identification and characterization of the novel gene GhDBP2 encoding a DRE-binding protein from cotton (Gossypium hirsutum). J Plant Physiol 2008, 165:214-223.

21. Shukla RK, Raha S, Tripathi V, Chattopadhyay D: Expression of CAP2, an APETALA2-family transcription factor from chickpea, enhances growth and tolerance to dehydration and salt stress in transgenic tobacco. Plant Physiol 2006, 142:113-123.

22. Mizoi J, Ohori T, Moriwaki T, Kidokoro S, Todaka D, Maruyama K, Kusakabe K, Osakabe Y, Shinozaki K, Yamaguchi-Shinozaki K: GmDREB2A;2, a canonical dehydration-responsive element-binding protein2-type transcription factor in soybean, is posttranslationally regulated and mediates dehydration-responsive element-dependent gene expression. Plant Physiol 2013, 161:346-361.

23. Xu ZS, Ni ZY, Li ZY, Li LC, Chen M, Gao DY, Yu XD, Liu P, Ma YZ: Isolation and functional characterization of HvDREB1-a gene encoding a dehydration-responsive element binding protein in Hordeum vulgare. J Plant Res 2009, 122:121-130.

24. Liu Q, Kasuga M, Sakuma Y, Abe H, Miura S, Yamaguchi-Shinozaki K, Shinozaki K: Two transcription factors, DREB1 and DREB2, with an EREBP/AP2 DNA binding domain separate two cellular signal transduction pathways in drought- and low-temperature-responsive gene expression, respectively, in Arabidopsis. Plant Cell 1998, 10:1391-1406.

25. Xiao BZ, Chen X, Xiang CB, Tang N, Zhang QF, Xiong LZ: Evaluation of seven function-known candidate genes for their effects on improving drought resistance of transgenic rice under field conditions. Mol Plant 2009, 2:73-83.

26. Yang S, Vanderbeld B, Wan J, Huang Y: Narrowing down the targets: towards successful genetic engineering of drought-tolerant crops. Mol Plant 2010, 3:469-490.
27. Ito Y, Katsura K, Maruyama K, Taji T, Kobayashi M, Seki M, Shinozaki K, Yamaguchi-Shinozaki K: Functional analysis of rice DREB1/CBF-type transcription factors involved in cold-responsive gene expression in transgenic rice. Plant Cell Physiol 2006, 47:141-153.

28. Osakabe Y, Kajita S, Osakabe K: Genetic engineering of woody plants: current and future targets in a stressful environment. Plant Physiol 2011, 142:05-117

29. Chen J, Xia X, Yin W: Expression profiling and functional characterization of a DREB2-type gene from Populus euphratica. Biochem Bioph Res Co 2009, 378:483-487.

30. Wang XM, Dong J, Liu Y, Gao HW: A novel dehydration-responsive element-binding protein from Caragana korshinskii is involved in the response to multiple abiotic stresses and enhances stress tolerance in transgenic tobacco. Plant Mol Biol Rep 2010, 28:664-675.

31. Zhang DY, Ma WB, Shi X, Wang JC, Wang XY: Distribution and bioecological characteristics of Eremosparton songoricum, a rare plant in gurbantunggut desert (in Chinese). J Desert Res 2008, 28:430-436.

32. Li XS, Yang HL, Zhang DY, Zhang YM, Wood AJ: Reference gene selection in the desert plant Eremosparton songoricum. Int J Mol Sci 2012, 13:6944-6963

33. Li HY, Li XS, Zhang DY, Liu HL, Guan KY: Effects of drought stress on the seed germination and early seedling growth of the endemic desert plant Eremosparton songoricum (fabaceae). Excli J 2013, 12:89-101.

34. Nakano T, Suzuki K, Fujimura T, Shinshi H: Genome-wide analysis of the ERF gene family in Arabidopsis and rice. Plant Physiol 2006, 140:411-432.

35. Li XP, Tian AG, Luo GZ, Gong ZZ, Zhang JS, Chen SY: Soybean DRE-binding transcription factors that are responsive to abiotic stresses. Theor Applied Genet 2005, 110:1355-1362.

36. Sakuma Y, Liu Q, Dubouzet JG, Abe H, Shinozaki K, Yamaguchi-Shinozaki K DNA-binding specificity of the ERF/AP2 domain of Arabidopsis DREBs, transcription factors involved in dehydration- and cold-inducible gene expression. Biochem Bioph Res Co 2002, 290:998-1009.

37. Gupta K, Agarwal PK, Reddy MK: SbDREB2A, An A-2 type DREB transcription factor from extreme halophyte Salicornia brachiata confers abiotic stress tolerance in Escherichia coli. Plant Cell Rep 2010, 29:1131-1137.

38. Matsukura S, Mizoi J, Yoshida T, Todaka D, Ito Y, Maruyama K, Shinozaki K, Yamaguchi-Shinozaki K: Comprehensive analysis of rice DREB2-type genes that encode transcription factors involved in the expression of abiotic stress-responsive genes. Mol Genet Genomics 2010, 283:185-196.

39. Sakuma Y, Maruyama K, Osakabe Y, Qin F, Seki M, Shinozaki K, YamaguchiShinozaki K: Functional analysis of an Arabidopsis transcription factor, DREB2A, involved in drought-responsive gene expression. Plant Cell 2006, 18:1292-1309.

40. Qin F, Kakimoto M, Sakuma Y, Maruyama K, Osakabe Y, Tran LS, Shinozaki K, Yamaguchi-Shinozaki K: Regulation and functional analysis of ZmDREB2A in response to drought and heat stresses in Zea mays L. Plant J 2007, 50:54-69.

41. Wang X, Chen X, Liu Y, Gao H, Wang Z, Sun G: CkDREB gene in Caragana korshinskii is involved in the regulation of stress response to multiple abiotic stresses as an AP2/EREBP transcription factor. Mol Biol Rep 2011, 38:2801-2811.

42. Shukla RK, Tripathi V, Jain D, Yadav RK, Chattopadhyay D: CAP2 enhances germination of transgenic tobacco seeds at high temperature and promotes heat stress tolerance in yeast. FEBS J 2009, 276:5252-5262

43. Agarwal P, Agarwal PK, Joshi AJ, Sopory SK, Reddy MK: Overexpression of PgDREB2A transcription factor enhances abiotic stress tolerance and activates downstream stress-responsive genes. Mol Biol Rep 2010, 37:1125-1135.

44. Sakuma Y, Maruyama K, Qin F, Osakabe Y, Shinozaki K, Yamaguchi-Shinozaki K: Dual function of an Arabidopsis transcription factor DREB2A in waterstress-responsive and heat-stress-responsive gene expression. P Natl Acad Sci USA 2006, 103:18822-18827.

45. Hodge A, Berta G, Doussan C, Merchan F, Crespi M: Plant root growth, architecture and function. Plant Soil 2009, 321:153-187.

46. Steudle E: Water uptake by roots: effects of water deficit. J Exp Bot 2000, 51:1531-1542

47. Verbruggen $N$, Hermanns C: Proline accumulation in plants: a review. Amino Acids 2008, 35:753-759.

48. Morgan JM: Osmoregulation and water stress in higher plants. Annu Rev Plant Physiol 1984, 35:299-319.

49. Cong L, Chai TY, Zhang YX: Characterization of the novel gene BjDREB1B encoding a DRE-binding transcription factor from Brassica juncea $\mathrm{L}$. Biochem Bioph Res Co 2008, 371:702-706. 
50. Zhao K, Shen X, Yuan H, Liu Y, Liao X, Wang Q, Liu L, Li F, Li T: Isolation and characterization of dehydration-responsive element-binding factor $2 \mathrm{C}$ (MsDREB2C) from Malus sieversii roem. Plant Cell Physiol 2013. in press. 10.1093/pcp/pct087.

51. Tang MJ, Sun JW, Liu Y, Chen F, Shen SH: Isolation and functional characterization of the JCERF gene, a putative AP2/EREBP domaincontaining transcription factor, in the woody oil plant Jatropha curcas. Plant Mol Biol 2007, 63:419-428.

52. Liu L, Zhu K, Yang Y, Wu J, Chen F, Yu D: Molecular cloning, expression profiling and trans-activation property studies of a DREB2-like gene from chrysanthemum (Dendranthema vestitum). J Plant Res 2008, 121:215-226.

53. Doyle JJ, Doyle JL: A rapid DNA isolation procedure for small quantities of fresh leaf tissue. Phytochem Bull 1987, 19:11-15.

54. Tamura K, Peterson D, Peterson N, Stecher G, Nei M, Kumar S: MEGA5: Molecular evolutionary genetics analysis using maximum likelihood, evolutionary distance, and maximum parsimony methods. Mol Biol Evol 2011, 28:2731-2739

55. Wood AJ, Duff RJD: The aldehyde dehydrogenase (ALDH) gene superfamily of the moss Physcomitrella patens and the algae Chlamydomonas reinhardtii \& Ostreococcus tauri. Bryologist 2009, 112:1-11.

56. Bailey TL, Elkan C: Fitting a Mixture Model by Expectation Maximization to Discover Motifs in Biopolymers, Proceedings of the Second International Conference on Intelligent Systems for Molecular Biology. Menlo Park, California: AAAI Press; 1994:28-36. 1994.

57. Bailey TL, Gribskov M: Combining evidence using p-values: application to sequence homology searches. Bioinformatics 1998, 14(1):48-54.

58. Teste MA, Duquenne M, Francois JM, Parrou JL: Validation of reference genes for quantitative expression analysis by real-time RT-PCR in Saccharomyces cerevisiae. BMC Mol Biol 2009, 10:99.

59. Wang BF, Wang YC, Zhang DW, Li HY, Yang CP: Verification of the resistance of a LEA gene from tamarix expression in Saccharomyces cerevisiae to abiotic stresses. J For Res 2008, 19(1):58-62.

60. Fisher DK, Guiltinan MJ: Rapid, efficient production of homozygous transgenic tobacco plants with Agrobacterium tumefaciens: a seed to seed protocol. Plant Mol Biol Rep 1995, 13:278-289.

61. Bates LS, Waldren RP, Teare ID: Rapid determination of free proline for water-stress studies. Plant Soil 1973, 39:205-207.

62. Peever TL, Higgins VJ: Electrolyte leakage, lipoxygenase, and lipid peroxidation induced in tomato leaf tissue by specific and nonspecific elicitors from Cladosporium fulvum. Plant Physiol 1989, 90:867-875.

63. Arnon DI: Copper enzymes in isolated chloroplasts. Polyphenoloxidase in Beta vulgaris. Plant Physiol 1949, 24:1-15.

doi:10.1186/1471-2229-14-44

Cite this article as: Li et al:: ESDREB2B, a novel truncated DREB2-type transcription factor in the desert legume Eremosparton songoricum, enhances tolerance to multiple abiotic stresses in yeast and transgenic tobacco. BMC Plant Biology 2014 14:44.

\section{Submit your next manuscript to BioMed Central and take full advantage of:}

- Convenient online submission

- Thorough peer review

- No space constraints or color figure charges

- Immediate publication on acceptance

- Inclusion in PubMed, CAS, Scopus and Google Scholar

- Research which is freely available for redistribution 